UT-STPD-4/00

BA-00-29

\title{
Monopoles, Axions and Intermediate Mass Dark Matter
}

\author{
G. Lazarides ${ }^{(1) *}$ and Q. Shafi ${ }^{(2) \dagger}$ \\ (1) Physics Division, School of Technology, Aristotle University of Thessaloniki, \\ Thessaloniki 540 06, Greece. \\ ${ }^{(2)}$ Bartol Research Institute, University of Delaware, Newark, DE 19716, USA.
}

\begin{abstract}
We present a solution to the cosmological problem encountered in (supersymmetric) grand unified theories due to copious monopole production at the end of hybrid inflation. By employing thermal inflation "driven" by the $U(1)$ axion symmetry, the superheavy monopole flux can be naturally suppressed to values that should be accessible to dedicated large scale experiments. The $U(1)$ axion symmetry also helps generate the right magnitude for the $\mu$ term of the minimal supersymmetric standard model. An important by-product is the predicted existence of stable or very longliving fermions possessing intermediate scale masses of order $10^{12} \mathrm{GeV}$. Their presence is required for implementing thermal inflation, and their stability is due to a $Z_{2}$ symmetry. They may constitute a sizable fraction of cold dark matter, and possibly help explain the ultra-high energy cosmic ray events. The rest of cold dark matter may consist of axions. Although our discussion is carried out within the framework of supersymmetric $S U(4)_{c} \times S U(2)_{L} \times S U(2)_{R}$, it can be extended to other grand unified gauge groups such as $S U(3)_{c} \times S U(3)_{L} \times S U(3)_{R}$ or $S O(10)$.
\end{abstract}

\footnotetext{
*lazaride@eng.auth.gr

${ }^{\dagger}$ shafi@bartol.udel.edu
} 
The great advantage of hybrid inflation [1] is that, in contrast to previous inflationary schemes, it can reproduce the observed temperature fluctuations of the cosmic microwave background radiation with natural values of the relevant coupling constant. Moreover, this inflationary scenario is almost automatically realized [2, 3] in supersymmetric (SUSY) grand unified theories (GUTs). However, in trying to apply it to GUTs which predict the existence of magnetic monopoles, a cosmological disaster is encountered. Hybrid inflation is terminated abruptly when the system reaches an instability point on the inflationary trajectory and is followed by a 'waterfall' regime during which the spontaneous breaking of the GUT gauge symmetry takes place. The appropriate Higgs fields develop their vacuum expectation values (vevs) starting from zero and they can end up at any point of the vacuum manifold with equal probability. As a consequence, monopoles are copiously produced [4] by the Kibble mechanism [5] leading to a cosmological catastrophe.

Possible solutions to this monopole problem have been proposed [4, 6]. They rely on introducing the leading non-renormalizable term in the standard superpotential [2] for hybrid inflation. (For different resolutions of the problem see Ref. [7].) In Ref. [4], the trilinear coupling of this standard superpotential was eliminated by a discrete symmetry and was replaced by the leading non-renormalizable term. The system, from the beginning of inflation, follows a particular valley and ends up at a particular point of the vacuum manifold. Thus, no monopoles can be produced. The inflationary trajectory possesses a classical inclination driving the inflaton towards the SUSY vacua and the termination of inflation is smooth. In Ref. [6], both the trilinear and the leading non-renormalizable couplings were kept revealing a quite different picture. The inflationary trajectory is classically flat and, thus, radiative corrections [3] are needed for driving the inflaton. The termination of inflation is abrupt. Nevertheless, there is no monopole production since the GUT gauge symmetry is already broken during inflation. Both models predict complete absence of monopoles which may be disappointing for the experimenters.

In this letter, we propose an alternative solution to the monopole problem of hybrid inflation which may yield a measurable monopole flux in our galaxy. The idea is to keep the original SUSY hybrid inflationary scenario unaltered and try to dilute the monopoles by invoking a subsequent thermal inflation [8,9] associated with an intermediate mass scale. (The main ideas underlying thermal inflation have been presented in Ref. [8]. The 
term thermal inflation was coined in Ref. [9], which further elaborated on the scheme.) It is then natural to identify this scale with the one at which the Peccei-Quinn (PQ) symmetry [10] is broken and also use [11] it for generating the $\mu$ term of the minimal supersymmetric standard model (MSSM). Although of much wider applicability, our mechanism is presented in the context of the SUSY Pati-Salam (PS) model [12], which is one the simplest unified schemes possessing [13] monopoles.

Our mechanism has an interesting by-product. In trying to make thermal inflation possible, we are led to the introduction of a number of superfields coupled to the field which breaks spontaneously the PQ symmetry. These fields possess intermediate scale masses, with the lightest ones being either stable or very long-living as a consequence of a $Z_{2}$ symmetry. Their fermionic components may constitute a sizable fraction of the cold dark matter in the universe, and possibly help explain [14] the ultra-high energy cosmic rays [15. Axions, of course, also may contribute to the cold dark matter fraction.

We consider the SUSY PS model [12] with gauge group $G_{P S}=S U(4)_{c} \times S U(2)_{L} \times$ $S U(2)_{R}$. The left-handed quark and lepton superfields are accommodated in the representations $F_{i}=(4,2,1), F_{i}^{c}=(\overline{4}, 1,2)$, where $i=1,2,3$ is the generation index. The two electroweak Higgs superfields belong to the representation $h=(1,2,2)$. The PS gauge group can be spontaneously broken to the standard model gauge group by a conjugate pair of Higgs superfields $H^{c}=(\overline{4}, 1,2), \bar{H}^{c}=(4,1,2)$ acquiring non-vanishing vevs along their right-handed neutrino directions. This can be achieved by introducing a gauge singlet superfield $S$ with two (renormalizable) superpotential terms: a term linear in $S$ and a trilinear coupling of $S$ to $H^{c}, \bar{H}^{c}$. The resulting scalar potential automatically possesses an in-built (classically) flat direction along which hybrid inflation can take place [2] with the system driven by an inclination from one-loop radiative corrections [3]. $G_{P S}$ is restored along the inflationary trajectory and breaks spontaneously only at the end of inflation when the system falls towards the SUSY vacua. This transition leads 四 to a cosmologically catastrophic copious production of doubly charged monopoles [13]. The monopoles could be diluted to an acceptable level if the primordial hybrid inflation is followed by thermal inflation [8.9]. This inflation, associated with an intermediate mass scale, is terminated at cosmic temperatures of the order of the electroweak scale and generates only a moderate number of e-foldings. 
Thermal inflation could be "driven" by the PQ symmetry which is spontaneously broken at an intermediate scale. Moreover, in Ref. [11], we showed that the PQ symmetry, which solves the strong $\mathrm{CP}$ problem, can also be used to generate the $\mu$ term of MSSM with the desired magnitude. More specifically, we introduced a pair of gauge singlet superfields $N, \bar{N}$ with non-zero PQ charges and a (non-renormalizable) superpotential coupling $N^{2} \bar{N}^{2}$. The resulting scalar potential of these fields, after soft SUSY breaking, possesses a non-trivial minimum which, under certain circumstances, is the absolute minimum, with $N, \bar{N}$ acquiring intermediate scale vevs, thereby breaking the PQ symmetry. The $\mu$ term is then generated via the superpotential coupling $N^{2} h^{2}$. The trivial extremum (at $N=\bar{N}=0$ ) turns out to be a local minimum separated from the PQ minimum by a sizable potential barrier. This situation persists at all cosmic temperatures after the primordial reheating which follows hybrid inflation, as has been shown [6] by including the one-loop temperature corrections to the potential. Thus, a successful transition from the trivial to the PQ minimum cannot be realized. We had to assume [6] that the system, after the primordial reheating, already emerges in the PQ vacuum. In other words, there is neither PQ transition nor thermal inflation in this case.

In order to make thermal inflation possible, we must turn the trivial local minimum of the zero-temperature potential into a saddle point. More specifically, the positive soft mass $^{2}$ term of $N$ should become negative. This can be achieved radiatively and requires strong couplings of $N$ to a number of superfields. To this end, we introduce $n$ superfields $D_{a}(a=1,2, \ldots, n)$ belonging to the representation $(6,1,1)$ of $G_{P S}$ with superpotential couplings $N D D$. However, these color (anti)triplets acquire intermediate scale masses after the PQ breaking, which could prevent the unification of the MSSM gauge coupling constants. To restore gauge unification, we include an equal number of superfields $H_{a}$ belonging to the $(1,2,2)$ representation with superpotential couplings $N H H$. Note that the negative mass ${ }^{2}$ term of $N$, which is successfully generated by invoking these extra superfields, gives rise to an additional problem. All the higher order terms in the scalar potential, which are derived from $N^{2} \bar{N}^{2}$ after soft SUSY breaking, involve both $N, \bar{N}$. Thus, these terms vanish along the $N$ direction, and the potential becomes unbounded below due to the negative mass ${ }^{2}$ term of $N$. Fortunately, there is a simple way out from this "runaway" problem. Replacing $N^{2} \bar{N}^{2}$ by $N^{3} \bar{N}$, we generate a $|N|^{6}$ term in the 
potential which prevents the runaway behavior along the $N$ direction.

We still need to include some extra couplings and superfields to obtain a phenomenologically viable scheme. In particular, we must introduce quartic superpotential couplings of $\bar{H}^{c}$ to $F_{i}^{c}$. These couplings generate intermediate scale masses for the right-handed neutrinos and, thus, seesaw masses for the light neutrinos. The inflaton then decays into right-handed neutrinos via the same couplings. Finally, in order to give superheavy masses to the down quark type components of $H^{c}, \bar{H}^{c}$, we include [16] an $S U(4)_{c} 6$-plet superfield $G=(6,1,1)$ with superpotential couplings $G H^{c} H^{c}, G \bar{H}^{c} \bar{H}^{c}$.

The superpotential of the model, which incorporates all the above couplings, is

$$
\begin{gathered}
W=\kappa S\left(\bar{H}^{c} H^{c}-M^{2}\right)+\lambda_{1} \frac{N^{2} h^{2}}{m_{P}}+\lambda_{2} \frac{N^{3} \bar{N}}{m_{P}}+\alpha_{a} N D_{a} D_{a}+\beta_{a} N H_{a} H_{a} \\
+\lambda_{i j} F_{i}^{c} F_{j} h+\gamma_{i j} \frac{\bar{H}^{c} \bar{H}^{c}}{m_{P}} F_{i}^{c} F_{j}^{c}+a G H^{c} H^{c}+b G \bar{H}^{c} \bar{H}^{c},
\end{gathered}
$$

where $M$ is a superheavy scale and $m_{P}=M_{P} / \sqrt{8 \pi} \approx 2.44 \times 10^{18} \mathrm{GeV}$ is the reduced Planck mass. Here, we chose a basis in the $D_{a}, H_{a}$ space where the coupling constant matrices $\alpha$ and $\beta$ are diagonal. Assuming that, at a more fundamental level, the $D$ 's and $H$ 's originate from $S O(10) 10$-plets, we can obtain the restriction $\alpha_{a}=\beta_{a}(a=1,2, \ldots, n)$. Note that $M, \kappa, \lambda_{1,2}, \alpha_{a}, \beta_{a}, a$ and $b$ can be made positive by field redefinitions.

In addition to $G_{P S}$, the superpotential in Eq.(1) possesses two continuous global (anomalous) symmetries, namely a $\mathrm{R}$ symmetry $U(1)_{R}$ and a $\mathrm{PQ}$ symmetry $U(1)_{P Q}$. The $\mathrm{R}$ and $\mathrm{PQ}$ charges of the superfields are assigned as follows:

$$
\begin{gathered}
R: H^{c}(0), \bar{H}^{c}(0), S(4), G(4), D(1), H(1), F(2), F^{c}(2), N(2), \bar{N}(-2), h(0) ; \\
P Q: H^{c}(0), \bar{H}^{c}(0), S(0), G(0), D(1), H(1), F(-2), F^{c}(0), N(-2), \bar{N}(6), h(2) .
\end{gathered}
$$

Note that the $\mathrm{R}$ charge of $W$ is 4 . Although it is not necessary, we also impose, for simplicity, a discrete $Z_{2}^{m p}$ symmetry ("matter parity"), under which $F, F^{c}$ change sign. Additional superpotential terms allowed by the symmetries of the model are

$$
F F H^{c} H^{c} N \bar{N}, F F H^{c} H^{c} h^{2}, F F \bar{H}^{c} \bar{H}^{c} N \bar{N}, F F \bar{H}^{c} \bar{H}^{c} h^{2}, F^{c} F^{c} H^{c} H^{c},
$$

modulo arbitrary multiplications by non-negative powers of the combinations $H^{c} \bar{H}^{c}$,

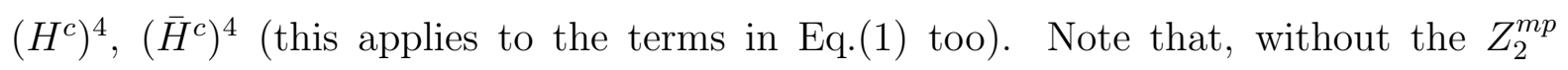
symmetry, the coupling $D H F \bar{H}^{c}$ would also be present in Eq.(3). 
Instanton and soft SUSY breaking effects explicitly break $U(1)_{R} \times U(1)_{P Q}$ to a discrete subgroup. It is then important to ensure that this subgroup is not spontaneously broken by the vevs of $N, \bar{N}$ since otherwise cosmologically disastrous domain walls will be produced in the PQ transition. This requirement implies that the number $n$ of $D$ 's and $H$ 's must be 5 or 7 . Moreover, in both these cases, the subgroup of $U(1)_{R} \times U(1)_{P Q}$ left unbroken by instantons and SUSY breaking coincides with the one left unbroken by $\langle N\rangle,\langle\bar{N}\rangle$, and is a $Z_{2} \times Z_{2}$ generated by $\left(e^{i \pi / 2}, e^{i \pi / 2}\right)$ and $\left(1, e^{i \pi}\right)$. (Note that the element $\left(e^{i \pi}, e^{i \pi}\right)$ is equivalent to the identity element since it leaves unaltered all the superfields of the theory.) Combining appropriately these $Z_{2}$ 's with $Z_{2}^{m p}$ and the $Z_{2}$ center of $S U(2)_{L}$, we obtain two equivalent $Z_{2}$ 's under which the $D$ 's or the $H$ 's change sign. It is interesting to note that, even if only $N$ develops a non-zero vev (see below), absence of domain walls still implies $n=5$ or 7 . The soft SUSY breaking terms respect the symmetry $Z_{2} \times U(1)_{P Q}$, where the non-anomalous $Z_{2}$ is generated by $\left(e^{i \pi / 2}, e^{i \pi / 2}\right)$. It is then obvious that further breaking of $U(1)_{P Q}$ to $Z_{2}$ by the vev of $N$ can solve the strong CP problem. Thus, we have the option to keep $\langle\bar{N}\rangle=0$. The symmetries which survive after instanton effects, in this case, are the same as in the $\langle\bar{N}\rangle \neq 0$ case.

Let us note that baryon and lepton number violations arise from the last three superpotential terms in Eq.(3), the last two couplings in Eq.(1) and the combinations $\left(H^{c}\right)^{4}$, $\left(\bar{H}^{c}\right)^{4}$, in complete analogy with Ref. [6]. The proton is practically stable.

The part of the tree-level scalar potential which is relevant for the PQ (and R symmetry) breaking can be derived from the superpotential term $N^{3} \bar{N}$ and, after soft SUSY breaking mediated by minimal supergravity, is given by (compare with Ref. [11)

$$
V_{P Q}=m_{3 / 2}^{2}\left(|N|^{2}+|\bar{N}|^{2}+\lambda_{2}^{2} \frac{|N|^{4}}{\left(m_{3 / 2} m_{P}\right)^{2}}\left(|N|^{2}+9|\bar{N}|^{2}\right)-|A| \lambda_{2} \frac{|N||\bar{N}|}{m_{3 / 2} m_{P}}|N|^{2}\right)
$$

where $m_{3 / 2}$ is the gravitino mass and $A$ is the dimensionless coefficient of the soft SUSY breaking term which corresponds to the superpotential coupling $N^{3} \bar{N}$. Here, the phases $\varphi, \theta$ and $\bar{\theta}$ of $A, N$ and $\bar{N}$ are taken to satisfy the relation $\varphi+3 \theta+\bar{\theta}=\pi$ which minimizes the potential for given values of $|N|,|\bar{N}|$.

As a consequence of the couplings of $N$ to the $D$ 's and $H^{\prime}$ 's, its mass ${ }^{2}$ in Eq.(4) can easily turn radiatively to negative values at lower energy scales (see also Ref. [17]). To see this, we consider the one-loop renormalization group equations for the soft masses 
$m_{N}, m_{D}$ and $m_{H}$ of the scalar fields $N, D$ and $H$ (see Ref. [18]):

$$
\begin{gathered}
\mu \frac{d m_{N}^{2}}{d \mu}=3 n Y_{D}\left(2 m_{D}^{2}+m_{N}^{2}\right)+2 n Y_{H}\left(2 m_{H}^{2}+m_{N}^{2}\right), \\
\mu \frac{d m_{D}^{2}}{d \mu}=Y_{D}\left(2 m_{D}^{2}+m_{N}^{2}\right), \mu \frac{d m_{H}^{2}}{d \mu}=Y_{H}\left(2 m_{H}^{2}+m_{N}^{2}\right),
\end{gathered}
$$

where $\mu$ is the running energy scale, $Y_{D}=\alpha^{2} / 2 \pi^{2}, Y_{H}=\beta^{2} / 2 \pi^{2}$, with $\alpha=\alpha_{a}, \beta=\beta_{a}$ $(a=1,2, \ldots, n)$. Here we take universal "asymptotic" soft scalar masses $\left(m_{N}=m_{D}=\right.$ $m_{H}=m_{3 / 2}$ at the GUT scale) and assume, for simplicity, that all the $\alpha$ 's ( $\beta$ 's) are equal and, thus, all the $D$ 's ( $H$ 's) have the same soft mass at all scales. This system of equations possesses [18] a non-trivial fixed point given by

$$
m_{N}^{2}=-2 m_{D}^{2}=-2 m_{H}^{2}=-\frac{2(5 n-1)}{5 n+2} m_{3 / 2}^{2},
$$

and admits the solution

$$
\begin{aligned}
& \frac{m_{N}^{2}}{m_{3 / 2}^{2}}=-\frac{2(5 n-1)}{5 n+2}+\frac{15 n}{5 n+2}\left(\frac{\mu}{M_{G}}\right)^{(5 n+2) Y}, \\
& \frac{m_{D}^{2}}{m_{3 / 2}^{2}}=\frac{m_{H}^{2}}{m_{3 / 2}^{2}}=\frac{5 n-1}{5 n+2}+\frac{3}{5 n+2}\left(\frac{\mu}{M_{G}}\right)^{(5 n+2) Y} .
\end{aligned}
$$

For simplicity, we further assumed that $\alpha=\beta$ (and, thus, $Y_{D}=Y_{H}=Y$ ), and we ignored the running of these coupling constants. Taking strong Yukawa couplings $\alpha=\beta=1$, we see that, already at $\mu / M_{G} \sim 10^{-2}$, the second terms in the right hand sides of the equalities in Eq.(7) are much smaller than $1 \%$ of the first terms. Thus, after the primordial reheating, the soft masses can be taken equal to their fixed point values.

The radiatively improved zero-temperature scalar potential is given by Eq.(四) with the $|N|^{2}$ term acquiring an extra negative factor $-2(5 n-1) /(5 n+2)$. The trivial (local) minimum of this potential (at $N=\bar{N}=0$ ) then becomes a saddle point and the absolute minimum necessarily lies at a non-zero value of $N$. It should be noted, however, that $\bar{N}$ is also non-zero at the absolute minimum only if $A \neq 0$. To simplify the presentation, we choose $A=0$. The absolute minimum of the potential then lies at

$$
|\langle N\rangle| \equiv \frac{f_{a}}{\sqrt{2}}=\left(\frac{10 n-2}{15 n+6}\right)^{1 / 4} \frac{\left(m_{3 / 2} m_{P}\right)^{1 / 2}}{\lambda_{2}^{1 / 2}},|\langle\bar{N}\rangle|=0 .
$$

The constant energy density carried by the trivial vacuum is given by 


$$
V_{0}=2\left(\frac{10 n-2}{15 n+6}\right)^{3 / 2} \frac{m_{3 / 2}^{3} m_{P}}{\lambda_{2}},
$$

and is responsible for driving thermal inflation.

The one-loop temperature corrections to the PQ potential can be calculated by employing the formalism of Ref. [19]. The absolute minimum of the resulting temperaturedependent effective potential at high cosmic temperatures $T$ lies at $N=\bar{N}=0$. So, after the primordial reheating following hybrid inflation, the system emerges in the trivial vacuum. The temperature correction to the mass ${ }^{2}$ term of the field $N$ is

$$
\delta V_{T} \approx n T^{2}\left(3 \alpha^{2}+2 \beta^{2}\right)|N|^{2}
$$

for $|N| \ll T$. Here, we considered only the main contributions which originate from loops with the superfields $D$ or $H$ circulating. As $T$ approaches the critical temperature

$$
T_{c} \approx \sqrt{\frac{10 n-2}{\left(5 n^{2}+2 n\right)\left(3 \alpha^{2}+2 \beta^{2}\right)}} m_{3 / 2}
$$

the potential barrier separating the trivial and PQ vacua becomes vanishingly small and the PQ transition takes place close to $T_{c}$ [8]. For a period preceding this transition, the energy density of the trivial vacuum dominates over the radiation energy density and the universe undergoes a mild inflationary phase known as thermal inflation [8.9]. This is terminated at the PQ transition where the system enters into an oscillatory phase about the PQ minimum. The coherently oscillating field $N$ (thermal inflaton) with mass $m_{\text {infl }}=2[(5 n-1) /(5 n+2)]^{1 / 2} m_{3 / 2}$ eventually decays, via the superpotential coupling $N^{2} h^{2}$, to a pair of Higgsinos thereby reheating the universe. Of course, this decay is possible only if the Higgsino mass $\mu=\lambda_{1} f_{a}^{2} / m_{P}$ is smaller than $m_{\text {infl }} / 2$. The decay width is $\Gamma \approx\left(2 \lambda_{1}^{2} f_{a}^{2} / \pi m_{P}^{2}\right)\left(1-\epsilon^{2}\right)^{1 / 2} m_{\text {infl }}$ where $\epsilon=2 \mu / m_{\text {infl }}(0<\epsilon<1)$. The maximal reheat temperature is achieved at $\epsilon^{2}=2 / 3$, which maximizes $\Gamma$, and is given by

$$
T_{r} \approx \frac{30^{1 / 4} \lambda_{1} f_{a} m_{i n f l}^{1 / 2}}{\pi g_{*}^{1 / 4}\left(T_{r}\right) m_{P}^{1 / 2}}
$$

where $g_{*}\left(T_{r}\right) \approx 89.75$ is the effective number of degrees of freedom at the reheat temperature $T_{r} \sim \mathrm{GeV}$ (see below).

The relative monopole number density $n_{M} / s\left(n_{M}\right.$ is the number density of monopoles and $s$ the entropy density) remains [20] essentially constant for temperatures between the 
primordial reheat temperature $T_{R} \lesssim 10^{12} \mathrm{GeV}$ (see below) and the critical temperature $T_{c}$, where the vacuum energy density $V_{0}$ is transferred to the oscillating inflaton field. The initial number density of thermal inflatons is $n_{\text {infl }} \approx V_{0} / m_{\text {infl }}$. So, at $T_{c}$,

$$
\frac{n_{M}}{n_{\text {infl }}} \approx \frac{n_{M}}{s} \frac{2 \pi^{2} g_{*}\left(T_{c}\right) T_{c}^{3} m_{\text {infl }}}{45 V_{0}}
$$

where $g_{*}\left(T_{c}\right) \approx 105.75+35 n / 2$, for $T_{c} \sim 70 \mathrm{GeV}$ (see below), is the effective number of degrees of freedom just before the PQ transition. Note that, for temperatures between $T_{R}$ and $T_{c}$, the fermionic components of the $D$ 's and $H$ 's are massless. The ratio in Eq.(13) remains [21] practically unaltered until $T_{r}$, where $n_{M} / s=\left(n_{M} / n_{\text {infl }}\right)\left(n_{\text {infl }} / s\right)$ with $n_{\text {infl }} / s \approx 3 T_{r} / 4 m_{\text {infl }}$ in the instantaneous inflaton decay approximation. Combining this with Eq.(13), one obtains the dilution factor of the relative monopole number density during the $\mathrm{PQ}$ transition and subsequent reheating:

$$
\left(\frac{n_{M}}{s}\right)\left(T_{r}\right) \approx\left(\frac{n_{M}}{s}\right)\left(T_{R}\right) \frac{\pi^{2} g_{*}\left(T_{c}\right) T_{c}^{3} T_{r}}{30 V_{0}} .
$$

At the primordial reheating, we have $n_{M} / s \approx\left(n_{M} / n_{I N F L}\right)\left(3 T_{R} / 4 m_{I N F L}\right)$, where $m_{I N F L}=\sqrt{2} \kappa M\left(n_{I N F L}\right)$ is [22] the inflaton mass (number density) for hybrid inflation. The ratio $n_{M} / n_{I N F L}$ remains [21] essentially unaltered after the production of monopoles at the end of hybrid inflation until $T_{R}$. The initial number density of magnetic monopoles can be estimated by the Kibble mechanism [5] which gives $n_{M} \approx(3 p / 4 \pi) m_{I N F L}^{3}$, where $p \sim 1 / 10$ is a geometric factor. This, together with $n_{I N F L} \approx V_{I N F L} / m_{I N F L}$, where $V_{I N F L}=\kappa^{2} M^{4}$ is [3] the constant energy density driving hybrid inflation, yields $n_{M} / n_{I N F L} \approx 3 p m_{I N F L}^{4} / 4 \pi V_{I N F L}$ and, thus,

$$
\left(\frac{n_{M}}{s}\right)\left(T_{R}\right) \approx \frac{9 \sqrt{2} p \kappa T_{R}}{8 \pi M} .
$$

The fraction of the inflationary energy density which goes into magnetic monopoles right after the end of hybrid inflation is $\approx 3 p m_{I N F L}^{3} m_{M} / V_{I N F L}=3 p \kappa m_{M} / \sqrt{2} \pi M \ll 1$, for all relevant $\kappa$ 's. Here $m_{M} \approx 4 \pi M / g_{G}$ is the monopole mass, with $g_{G}$ being the GUT gauge coupling constant. The final $n_{M} / s$ can then be found from Eqs.(14) and (15).

The primordial inflaton decays into a pair of right-handed neutrino superfields $\nu^{c}$ with mass $M_{\nu^{c}}=\eta m_{I N F L} / 2(0<\eta<1)$ via the superpotential coupling $\bar{H}^{c} \bar{H}^{c} F^{c} F^{c}$. 
The decay width is $\approx(1 / 8 \pi)\left(M_{\nu^{c}} / M\right)^{2}\left(1-\eta^{2}\right)^{1 / 2} m_{I N F L}$. Note that $M_{\nu^{c}}$ also originates from the coupling $\bar{H}^{c} \bar{H}^{c} F^{c} F^{c}$ and, thus, cannot be bigger than about $2 M^{2} / m_{P}$. The primordial reheat temperature is given by

$$
T_{R} \approx \frac{3^{1 / 2} 5^{1 / 4} \kappa^{1 / 2} m_{P}^{1 / 2} M_{\nu^{c}}\left(1-\eta^{2}\right)^{1 / 4}}{2 \sqrt{2} \pi g_{*}^{1 / 4}\left(T_{R}\right) M^{1 / 2}},
$$

where $g_{*}\left(T_{R}\right) \approx 234.25+75 n / 2$ since, at $T_{R}$, the bosonic $D$ 's and $H$ 's are still massless.

For each $\kappa$, the value of $M$ can be found by simultaneously solving Eqs.(2) and (5) of Ref. [23] with their right hand sides divided by an extra factor of 2 due to the fact that $H^{c}\left(\bar{H}^{c}\right)$ contains four $S U(2)_{R}$ doublets. We will take, for definiteness, $\kappa=4 \times 10^{-3}$ which gives $M \approx 9.57 \times 10^{15} \mathrm{GeV}$. With these values, hybrid inflation ends only when the inflaton field $S$ is infinitesimally close to the instability point (at $|S|=M$ ) of the inflationary trajectory, as one can easily deduce from the slow roll conditions. Moreover, our present horizon scale crosses outside the inflationary horizon at $|S| \approx 2.63 M$. From Eq.(16), we find that $T_{R}$ can take all the values up to about $10^{12} \mathrm{GeV}$. The maximal $T_{R}$ is obtained at $\eta^{2}=2 / 3$ which maximizes the decay width of the primordial inflaton. The corresponding value of $M_{\nu^{c}}$ turns out to be much smaller than $2 M^{2} / m_{P}$. It is important to note that the stringent gravitino constraint [24] on $T_{R}\left(\lesssim 10^{9} \mathrm{GeV}\right)$ is alleviated here since the primordial gravitinos suffer considerable dilution during thermal inflation.

We will now estimate the present $D$ and $H$ particle abundance. Due to the two unbroken $Z_{2}$ 's under which the $D$ 's and $H$ 's change sign independently, these particles can only annihilate in pairs. The annihilation processes remain in thermal equilibrium at all temperatures between $T_{R}$ and $T_{c}$. Moreover, at temperatures higher than $T_{c}$, where the PQ (thermal) transition takes place, the fermionic $D$ 's and $H$ 's are massless. On the contrary, the scalar components of these fields acquire soft SUSY breaking and temperature-dependent masses. Consequently, for $m_{3 / 2} \gg T_{c}$ (see below), the number density of these bosons at temperatures close to $T_{c}$ is suppressed, and we thus ignore them. The number density of the fermionic $D$ 's $\left(H\right.$ 's), for temperatures just above $T_{c}$, is $n_{D}\left(T_{c}\right) \approx 21 n \zeta(3) T_{c}^{3} / 2 \pi^{2}\left(n_{H}\left(T_{c}\right) \approx 14 n \zeta(3) T_{c}^{3} / 2 \pi^{2}\right)$, where $\zeta(3) \approx 1.2021$.

After the completion of thermal transition, the $D(H)$ superfields acquire intermediate scale masses $\sqrt{2} \alpha f_{a}\left(\sqrt{2} \beta f_{a}\right)$. Their total relative contribution to the energy density of the universe, immediately following the transition, is given by $\Omega_{D H}\left(T_{c}\right) \equiv$ 
$\rho_{D H}\left(T_{c}\right) / \rho_{\text {infl }}\left(T_{c}\right) \approx 7 n(3 \alpha+2 \beta) \zeta(3) f_{a} T_{c}^{3} / \sqrt{2} \pi^{2} V_{0} \ll 1$, where $\rho_{\text {infl }}$ is the energy density in thermal inflatons. $\Omega_{D H}$ remains essentially constant until $T_{r}$ since pair annihilation of the fermionic $D$ 's and $H$ 's is frozen already at $T_{c}$ where these fermions acquire their intermediate scale masses. Between $T_{r}$ and the equidensity temperature $T_{e q} \approx 2.5 \times 10^{-9} \mathrm{GeV}$ (for present value of the Hubble constant $H_{0} \approx 65 \mathrm{~km} / \mathrm{s} \mathrm{Mpc}$ ), where matter and radiation have equal energy densities, $\Omega_{D H}$ is enhanced by a factor $T_{r} / T_{e q}$ and remains practically unaltered thereafter. So the present abundance of these particles is

$$
\Omega_{D H} \approx \frac{7 n(3 \alpha+2 \beta) \zeta(3)}{\sqrt{2} \pi^{2}} \frac{f_{a} T_{c}^{3}}{V_{0}} \frac{T_{r}}{T_{e q}} .
$$

This can easily be of order unity so that the $D$ and $H$ fermions with intermediate scale masses constitute a sizable fraction of the cold dark matter in the present universe.

We are now ready to proceed to a numerical example. We choose the gravitino mass $m_{3 / 2}=300 \mathrm{GeV}$ and the number of $D$ 's and $H$ 's $n=7$. The thermal inflaton mass is then $m_{\text {infl }} \approx 575 \mathrm{GeV}$ and the Higgsino mass $\mu \approx 235 \mathrm{GeV}$ (recall that we take $\epsilon^{2}=2 / 3$ ). The critical temperature for the PQ transition can be evaluated from Eq.(11) and turns out to be $T_{c} \approx 69 \mathrm{GeV}$ for coupling constants $\alpha=\beta=1$. We further take $\lambda_{2}=2 \times 10^{-3}$. From Eqs.(8) and (9), we then obtain the axion decay constant $f_{a} \approx 7.57 \times 10^{11} \mathrm{GeV}$ and the vacuum energy density which drives thermal inflation $V_{0} \approx 3.16 \times 10^{28} \mathrm{GeV}^{4}$. The parameter $\lambda_{1}$ is evaluated from the Higgsino mass and comes out to be $\approx 10^{-3}$. Eq.(12) then yields $T_{r} \approx 2.81 \mathrm{GeV}$ for the reheat temperature after thermal inflation.

The present relative monopole number density is estimated from Eqs.(14) and (15). It turns out to be $n_{M} / s \approx 4.6 \times 10^{-41} T_{R}$ for the chosen value of $\kappa\left(=4 \times 10^{-3}\right)$. This implies that $T_{R}$ 's of order $10^{10} \mathrm{GeV}$, which can be naturally obtained from Eq.(16) by appropriately adjusting $M_{\nu^{c}}$ (or $\eta$ ), lead to $n_{M} / s \sim 10^{-30}$. This corresponds to the well-known Parker bound for the monopole flux in our galaxy derived from galactic magnetic field considerations. Needless to say that, by lowering $T_{R}$, one can easily reduce the predicted monopole flux by a couple of orders of magnitude below the Parker bound. However, this flux cannot be suppressed much further with natural (not too small) values of the coupling constants. In conclusion, we have shown that thermal inflation associated with the PQ symmetry and the $\mu$ term can naturally suppress the present flux of monopoles from SUSY hybrid inflation to values below but near the Parker 
bound. This flux should be possibly accessible to ongoing and future experiments.

The present abundance of the $D$ and $H$ fermions with masses $\approx 1.1 \times 10^{12} \mathrm{GeV}$ is found from Eq.(17) to be $\Omega_{D H} \approx 0.185$. These particles can therefore provide a considerable fraction of the cold dark matter. The rest can consist of axions which are also present in this scheme. The relic axion abundance $\Omega_{a}$ has been calculated in Ref. [25]. Assuming that the initial value of the axion field is about $0.55 f_{a}$, Eq.(13) of the first paper in this reference yields $\Omega_{a} \approx 0.115$ for $H_{0}=65 \mathrm{~km} / \mathrm{s} \mathrm{Mpc}$ and $f_{a}=7.57 \times 10^{11} \mathrm{GeV}$. We see that one can naturally obtain a cold dark matter component in the universe consisting of both axions and intermediate scale mass fermions with total energy density equal to about $30 \%$ of its critical density, consistent with recent observations [26.27]. It should be clear that, by appropriately choosing the values of the parameters, one can easily adjust not only the total cold dark matter abundance but also its composition of axions and intermediate scale mass fermions. The present numbers only serve as an example.

As explained, the $D$ and $H$ fermions are stable due to the two $Z_{2}$ remnants of $U(1)_{R} \times U(1)_{P Q}$. Their stability is absolute if these $U(1)$ 's are exact symmetries of the superpotential to all orders. However, as has been shown [28], global $U(1)$ 's may be present as effective rather than exact symmetries. Indeed, some of the discrete symmetries which normally emerge from the underlying string theory can effectively behave as continuous symmetries. These continuous symmetries are expected to be explicitly broken by some higher order operators in the superpotential which are allowed by the underlying discrete symmetries. If the order of these operators is adequately high, they do not affect our scheme except that they may provide highly suppressed Yukawa couplings for the decay of the $D$ 's and/or the $H$ 's by violating either or both the $Z_{2}$ 's. Such couplings could be $D F F, D F^{c} F^{c}$ or $H F F^{c}$ with coefficients suppressed by $\left(f_{a} / m_{P}\right)^{5}$ leading to a lifetime $\sim 10^{22}$ years. The long-living $D$ 's and $H$ 's with masses $\sim 10^{12} \mathrm{GeV}$ may then provide an explanation [14] of the recently observed [15] ultra-high energy cosmic ray events. Note that these intermediate scale mass particles were introduced for making thermal inflation possible, and thus provide a mechanism for diluting the monopoles. Their role in dark matter and cosmic rays is an extra bonus!

It is generally difficult to generate the baryon asymmetry of the universe in models with a low reheat temperature such as our scheme. Any pre-existing baryon (or lepton) 
asymmetry is utterly diluted by thermal inflation. Moreover, after the subsequent reheating, the universe is too cold to allow baryon number violation and out-of-equilibrium conditions. Baryogenesis mechanisms which may be applicable here have been discussed in Refs. [29, 30]. The latter uses the fact [31] that the oscillating (thermal) inflaton does not decay instantaneously at $T_{r}$. It rather follows the usual exponential decay law. The "new radiation", which is so produced, reaches a maximum temperature which is much higher than the electroweak scale. This radiation then gradually cools down and, finally, dominates the energy density at $T_{r}$. During this process, a lepton asymmetry can be generated via the Affleck-Dine (AD) mechanism [32]. The decay of the AD condensate is plasma blocked at temperatures higher than its frequency of oscillations which is expected to be of the order of the electroweak scale. Actually, this condensate decays at a temperature $T_{*} \sim M_{W}$, generating a lepton asymmetry of order unity (or smaller), provided that its energy density at $T_{*}$ is comparable to (or smaller than) the "new radiation" energy density. A fraction of this asymmetry is then immediately converted into baryon asymmetry by the electroweak sphalerons. From $T_{*}$ until $T_{r}$, the baryon asymmetry acquires [30] a dilution factor $\left(T_{r} / T_{*}\right)^{5}$ and remains constant thereafter. It is clear that this scenario can easily lead to an adequate baryogenesis in our scheme.

In summary, we considered the cosmological problem arising when hybrid inflation is applied to (SUSY) GUTs which predict magnetic monopoles. This problem is due to the copious monopole production at the end of inflation. We showed that the monopole flux can be naturally reduced to values below but near the Parker bound by invoking thermal inflation "driven" by the PQ symmetry, which also generates the $\mu$ term of MSSM. This flux may be accessible to ongoing and future experiments. Although our mechanism was presented within the SUSY PS model, its applicability is much wider. An interesting by-product is the presence of intermediate scale mass fermions and axions in the cold dark matter of the universe. These fermions, which were introduced for making thermal inflation possible, may explain the recently observed ultra-high energy cosmic rays.

We thank A. Riotto for useful discussions. This work was supported by the European Union under TMR contract number ERBFMRX-CT96-0090, the DOE under grant number DE-FG02-91ER40626, and the NSF under subcontract PHY-9800748. 


\section{REFERENCES}

[1] A. D. Linde, Phys. Rev. D49 (1994) 748.

[2] E. J. Copeland, A. R. Liddle, D. H. Lyth, E. D. Stewart and D. Wands, Phys. Rev. D49 (1994) 6410.

[3] G. Dvali, R. Schaefer and Q. Shafi, Phys. Rev. Lett. 73 (1994) 1886.

[4] G. Lazarides and C. Panagiotakopoulos, Phys. Rev. D52 (1995) 559.

[5] T. W. B. Kibble, J. Phys. A9 (1976) 1387; Phys. Rev. D67 (1980) 183.

[6] R. Jeannerot, S. Khalil, G. Lazarides and Q. Shafi, hep-ph/0002151.

[7] G. Dvali, H. Liu and T. Vachaspati, Phys. Rev. Lett. 80 (1998) 2281; G. Dvali, L. M. Krauss and H. Liu, hep-ph/9707456.

[8] G. Lazarides, C. Panagiotakopoulos and Q. Shafi, Phys. Rev. Lett. 56 (1986) 557; G. Lazarides and Q. Shafi, Nucl. Phys. B392 (1993) 61.

[9] D. H. Lyth and E. D. Stewart, Phys. Rev. Lett. 75 (1995) 201.

[10] R. Peccei and H. Quinn, Phys. Rev. Lett. 38 (1977) 1440; S. Weinberg, Phys. Rev. Lett. 40 (1978) 223; F. Wilczek, Phys. Rev. Lett. 40 (1978) 279.

[11] G. Lazarides and Q. Shafi, Phys. Rev. D58 (1998) 071702.

[12] J. C. Pati and A. Salam, Phys. Rev. D10 (1974) 275.

[13] G. Lazarides, M. Magg and Q. Shafi, Phys. Lett. 97B (1980) 87.

[14] V. Berezinsky, M. Kachelriess and A. Vilenkin, Phys. Rev. Lett. 79 (1997) 4302; M. Birkel and S. Sarkar, Astropart. Phys. 9 (1998) 297; V. A. Kuzmin and V. A. Rubakov, Phys. Atom. Nucl. 61 (1998) 1028; O. E. Kalashev, V. A. Kuzmin and D. V. Semikoz, astro-ph/9911035; V. A. Kuzmin and I. I. Tkachev, Phys. Rep. 320 (1999) 199.

[15] D. J. Bird et al., Phys. Rev. Lett. 71 (1993) 3401; Astrophys. J. 424 (1994) 491; ibid. 441 (1995) 144; N. Hayashida et al., Phys. Rev. Lett. 73 (1994) 3491; S. 
Yoshida et al., Astropart. Phys. 3 (1995) 105; M. Takeda et al., Phys. Rev. Lett. 81 (1998) 1163.

[16] I. Antoniadis and G. K. Leontaris, Phys. Lett. B216 (1989) 333.

[17] K. Choi, E. J. Chun and J. E. Kim, Phys. Lett. B403 (1997) 209.

[18] M. Mangano, Z. Phys. C28 (1985) 613.

[19] L. Dolan and R. Jackiw, Phys. Rev. D9 (1974) 3320; S. Weinberg, Phys. Rev. D9 (1974) 3357.

[20] J. P. Preskill, Phys. Rev. Lett. 43 (1979) 1365.

[21] G. Lazarides, C. Panagiotakopoulos and Q. Shafi, Phys. Rev. Lett. 58 (1987) 1707.

[22] G. Lazarides, R. K. Schaefer and Q. Shafi, Phys. Rev. D56 (1997) 1324.

[23] G. Lazarides and N. Vlachos, Phys. Lett. B459 (1999) 482.

[24] M. Yu. Khlopov and A. D. Linde, Phys. Lett. B138 (1984) 265 ; J. Ellis, J. E. Kim and D. Nanopoulos, Phys. Lett. B145 (1984) 181.

[25] J. Preskill, M. B. Wise and F. Wilczek, Phys. Lett. 120B (1983) 127; L. F. Abbott and P. Sikivie, Phys. Lett. 120B (1983) 133; M. Dine and W. Fischler, Phys. Lett. 120B (1983) 137.

[26] A. E. Evrard, Mon. Not. R. Astron. Soc. 292 (1997) 289.

[27] B. Schmidt et al., Astrophys. J. 507 (1998) 46; A. Riess et al., Astron. J. 116 (1998) 1009; S. Perlmutter et al., Bull. Am. Astron. Soc. 29 (1997) 1351.

[28] G. Lazarides, C. Panagiotakopoulos and Q. Shafi, Phys. Rev. Lett. 56 (1986) 432.

[29] L. M. Krauss and M. Trodden, Phys. Rev. Lett. 83 (1999) 1502.

[30] S. Davidson, M. Losada and A. Riotto, Phys. Rev. Lett. 84 (2000) 4284.

[31] R. J. Scherrer and M. S. Turner, Phys. Rev. D31 (1985) 681.

[32] I. Affleck and M. Dine, Nucl. Phys. B249 (1985) 361. 